\title{
Exponential Hedging and Entropic Penalties
}

Freddy Delbaen

Department of Mathematics

ETH Zürich

CH - 8092 Zürich

Switzerland

Thorsten Rheinländer

Department of Mathematics

ETH Zürich

CH - 8092 Zürich

Switzerland

Martin Schweizer*

LMU München

Mathematisches Institut

Theresienstraße 39

D - 80333 München

Germany
Peter Grandits

TU Wien

Finanz- und Versicherungsmathematik

Wiedner Hauptstraße 8-10/E105

A - 1040 Wien

Austria

Dominick Samperi

Decision Synergy

375 South End Avenue, Suite 8J

New York, NY 10280

USA

Christophe Stricker

Université de Franche-Comté

Laboratoire de Mathématiques, UMR 6623

16 Route de Gray

F - 25030 Besançon Cedex

France

\footnotetext{
* corresponding author
} 
Abstract: We solve the problem of hedging a contingent claim $B$ by maximizing the expected exponential utility of terminal net wealth for a locally bounded semimartingale $X$. We prove a duality relation between this problem and a dual problem for local martingale measures $Q$ for $X$ where we either minimize relative entropy minus a correction term involving $B$ or maximize the $Q$-price of $B$ subject to an entropic penalty term. Our result is robust in the sense that it holds for several choices of the space of hedging strategies. Applications include a new characterization of the minimal martingale measure and risk-averse asymptotics.

Key words: hedging, exponential utility, relative entropy, duality, minimal martingale measure, minimal entropy martingale measure, reverse Hölder inequalities

MSC 2000 Classification Numbers: 91B28, 60G48

JEL Classification Numbers: C61, G11, G13

(Mathematical Finance 12 (2002), 99-123)

This version: 20.10 .2001 


\section{Introduction}

Hedging contingent claims is an important issue in mathematical finance, and several criteria for finding optimal strategies have been used in the literature. In this paper, we solve this problem when the quality of a strategy is measured by the expected exponential utility of its final net wealth. More precisely, let the semimartingale $X$ model the discounted price processes of the primary assets available for trade. The process $V(c, \vartheta)=c+\int \vartheta d X$ then describes the wealth evolution of a self-financing strategy $(c, \vartheta)$ with initial capital $c$ and $\vartheta_{t}^{i}$ units of asset $i$ held at time $t$. For a contingent claim with payoff $B$ at time $T$, we want to

$$
\operatorname{maximize} E_{P}\left[1-e^{-\alpha\left(V_{T}(c, \vartheta)-B\right)}\right]
$$

over all $\vartheta$ from a suitable space $\Theta$ of hedging strategies, i.e., integrands for $X$.

In connection with the optimization problem (0.1), we are interested in two things. We want to find the optimal strategy $\vartheta^{*}$ that maximizes the expression in $(0.1)$ and we want to compute the value of (0.1), i.e., the maximal expected exponential utility. For the latter, we consider the dual problem where we

$$
\operatorname{minimize} 1-\exp \left(-H(Q \mid P)-\alpha c+\alpha E_{Q}[B]\right)
$$

over a suitable class $\mathbb{P}$ of local martingale measures $Q$ for $X$. The problem (0.2) can be interpreted in two ways: we minimize the relative entropy $H(Q \mid P)$ minus a correction term depending on $B$, or we maximize the $Q$-price $E_{Q}[B]$ of $B$ with an entropic penalty term.

At a formal level, the functionals in $(0.1)$ and $(0.2)$ can be obtained from each other via Legendre transforms. The first contribution of this paper is a proof that (0.1) and (0.2) are in fact dual problems and have the same value. We prove this for three different choices of $\Theta$ and thus establish in particular a robustness result for the duality between (0.1) and (0.2). Moreover, we show that the supremum in (0.1) is attained for two of the three choices of $\Theta$ and identify the optimal strategy in terms of the solution of (0.2). Similar duality results have recently and mostly independently been established in a number of papers. Put briefly, the major distinction of our paper is the fact that we robustly obtain the same duality between (0.1) and (0.2) for several different choices of $\Theta$. A more detailed comparison is given at the end of section 1.

A second contribution of our paper is a number of applications of the basic duality result. Most notably, we provide for continuous $X$ a new characterization of the minimal martingale measure $\widehat{P}$. We show that $\widehat{P}$ is the unique solution of the dual problem (0.2) for a specific choice of $B$ : finding the minimal martingale measure corresponds to hedging with exponential utility a multiple of the total mean-variance tradeoff of $X$. We also consider the totally risk-averse limiting case $\alpha \rightarrow \infty$ in (0.1) and prove that this leads to superreplication of $B$. 
The paper is structured as follows. Section 1 introduces our setup and gives the three duality theorems 1, 2 and 3 together with the preliminaries required for their formulation. In section 2 , we use a measure transformation to reduce the problem to the case $B \equiv 0$ and prove Theorem 1. In addition, we prepare the ground for proving Theorems 2 and 3 by establishing a number of auxiliary results of some independent interest. Section 3 contains the proofs of Theorems 2 and 3; the most technical part is an approximation argument showing that it is enough to consider strategies with a uniformly bounded wealth process. In the final section 4, we first present the above two applications to the minimal martingale measure and to risk-averse asymptotics. We then informally discuss the Markovian case and conclude with a partial exploration of the limitations of our results.

\section{The key duality}

In this section, we introduce our basic setup and formulate the central duality result in three different versions. Proofs, examples and applications are deferred to later sections.

We start with a probability space $(\Omega, \mathcal{F}, P)$, a time horizon $T \in(0, \infty]$ and a filtration $\mathbb{F}=\left(\mathcal{F}_{t}\right)_{0 \leq t \leq T}$ satisfying the usual conditions of right-continuity and completeness. Thus we can and do choose RCLL versions for all $(P, \mathbb{F})$-semimartingales. All our processes are defined on $[0, T]$ so that all martingales are uniformly integrable. We fix throughout the paper an $\mathbb{R}^{d}$-valued $(P, \mathbb{F})$-semimartingale $X=\left(X_{t}\right)_{0 \leq t \leq T}$ and think of this as the discounted prices of $d$ risky assets in a financial market comprising also a riskless asset with discounted price constant at 1 . A self-financing trading strategy is then determined by its initial capital $c \in \mathbb{R}$ and the numbers $\vartheta_{t}^{i}$ of shares of asset $i, i=1, \ldots, d$, held at time $t \in[0, T]$. Formally, $\vartheta$ is in $L(X)$, i.e., an $\mathbb{F}$-predictable $X$-integrable $\mathbb{R}^{d}$-valued process; the (real-valued) stochastic integral process $G(\vartheta):=\int \vartheta d X$ is then well-defined, and the value or wealth at time $t$ of a strategy $(c, \vartheta)$ is $V_{t}(c, \vartheta)=c+G_{t}(\vartheta), 0 \leq t \leq T$. To exclude arbitrage opportunities, one has to impose additional conditions on $\vartheta$ and we denote by $\Theta$ a generic space of "permitted" trading strategies. Specific choices of $\Theta$ will be given later. Generic constants are denoted by $C$ and can vary from one appearance to the next. All expectations without subscript are under $P$.

Throughout this paper, we impose the standing assumption that

$X$ is locally bounded.

An absolutely continuous/equivalent local martingale measure (ALMM/ELMM) for $X$ is a probability measure $Q$ absolutely continuous/equivalent to $P$ such that $X$ is a local $(Q, \mathbb{F})$ martingale. We denote by $\mathbb{P}_{a}$ and $\mathbb{P}_{e}$ the spaces of all ALMMs and ELMMs respectively. Due to (1.1), a probability measure $Q$ is in $\mathbb{P}_{a}\left(\mathbb{P}_{e}\right)$ if and only if $Q \ll P(Q \approx P)$ and 
$E_{Q}\left[h^{\operatorname{tr}}\left(X_{\tau}-X_{\sigma}\right)\right]=0$ for all stopping times $\sigma \leq \tau \leq T$ such that $X^{\tau}$ is bounded and for all $\mathbb{R}^{d}$-valued bounded $\mathcal{F}_{\sigma}$-measurable random variables $h$. In analogy to $\Theta$, we denote by $\mathbb{P}$ a generic space of "relevant" ALMMs; specific choices will be discussed later. Finally, a contingent claim is an $\mathcal{F}_{T}$-measurable random variable $B$; it describes the net payoff at time $T$ of some financial instrument.

Our goal in this paper is to formulate and prove a duality result of the form

$$
\begin{aligned}
\sup _{\vartheta \in \Theta} E\left[1-e^{-\alpha\left(c+G_{T}(\vartheta)-B\right)}\right] & =1-\exp \left(-\inf _{Q \in \mathbb{P}}\left(H(Q \mid P)+\alpha c-E_{Q}[\alpha B]\right)\right) \\
& =1-\exp \left(\alpha \sup _{Q \in \mathbb{P}}\left(E_{Q}[B]-c-\frac{1}{\alpha} H(Q \mid P)\right)\right)
\end{aligned}
$$

for a given contingent claim $B$, a given initial capital $c$ and a given risk aversion parameter $\alpha>0$. In (1.2),

$$
H(Q \mid P):= \begin{cases}E_{P}\left[\frac{d Q}{d P} \log \frac{d Q}{d P}\right] & \text { if } Q \ll P \\ +\infty & \text { otherwise }\end{cases}
$$

denotes the relative entropy of $Q$ with respect to $P$. Of course, precise conditions on $B, \Theta$ and $\mathbb{P}$ still need to be specified.

Note that the left-hand side of (1.2) is the value of the problem of maximizing expected exponential utility from net terminal wealth if one has inital capital $c$ and the obligation to pay out $B$ at the end. Thus our primal problem can be viewed as a combination of investment and hedging. In particular, we can interpret the resulting strategy $\vartheta^{*}$ as optimal hedging strategy for an exponential utility criterion. For the special case $B \equiv 0$, we recover a pure investment problem and we shall see below that the general case can be reduced to this by a measure transformation.

For the corresponding dual problem, the middle expression in (1.2) involves minimizing relative entropy minus a correction term (depending on the contingent claim $B$ ) over all ALMMs $Q \in \mathbb{P}$; this can be viewed as an optimality criterion for the choice of a martingale measure $Q$. The right-hand side corresponds to a penalized maximization of the $Q$-price of the contingent claim $B$ with a penalty term proportional to the relative entropy $H(Q \mid P)$. One might call this pricing with an entropic penalty.

Remark. Since exponential utility is a popular concept, it is not surprising that variants of the duality (1.2) have appeared before. A one-period discrete-time version of (1.2) for the special case $B \equiv 0$ was established in Ben-Tal (1985). For $\Omega$ finite, this was generalized to arbitrary $B$ by Samperi (1998) who also conjectured that the result is true for general multiperiod models with infinite $\Omega$. For arbitrary $\Omega$ and a discrete-time setup, Samperi (2000) considered the one-period case for general $B$ and Frittelli (2000a) briefly discussed the multiperiod case with $B \equiv 0$. Samperi (2000) explores the connections to model calibration 
problems and also provides some historical comments. A duality result of the type in (1.2) with a general utility function $U$ in a general semimartingale setting was also established in Kramkov/Schachermayer (1999), but their assumptions do not cover the case of exponential utility treated here. For comments on a number of more recent papers, we refer to the end of this section.

Returning to the problem of a rigorous formulation, the easier part in making (1.2) precise is the choice of conditions on $\mathbb{P}$ and $B$. The middle expression in (1.2) does not involve $\Theta$, but only $H(Q \mid P)-E_{Q}[\alpha B]$ which is to be minimized over $Q \in \mathbb{P} \subseteq \mathbb{P}_{a}$. This difference is undefined if both terms are $+\infty$ and so we should either assume that $B$ is bounded above (so that we can allow $\mathbb{P}=\mathbb{P}_{a}$ ) or ensure by choice of $\mathbb{P}$ that $H(Q \mid P)$ is finite. We opt for the latter because it gives us more freedom in the choice of $B$. Hence we choose

$$
\mathbb{P}_{f}:=\mathbb{P}_{f}(P):=\left\{Q \in \mathbb{P}_{a} \mid H(Q \mid P)<\infty\right\}
$$

(where $f$ indicates finite relative entropy) and we impose the natural assumption that

$$
\mathbb{P}_{f}(P) \neq \emptyset \text {, i.e., there exists some ALMM } Q \text { with } H(Q \mid P)<\infty \text {. }
$$

This has two very useful consequences. By Theorem 2.1 of Frittelli (2000a), (1.3) implies that there exists a unique $Q^{E}$ in $\mathbb{P}_{f}(P)$ that minimizes $H(Q \mid P)$ over all $Q \in \mathbb{P}_{f}(P)$. This $Q^{E} \ll P$ is called the minimal entropy martingale measure or minimal $P$-entropy martingale measure if we want to emphasize the reference measure. If we even have

$$
\mathbb{P}_{f}(P) \cap \mathbb{P}_{e} \neq \emptyset \text {, i.e., there exists some ELMM } Q \text { with } H(Q \mid P)<\infty
$$

then $Q^{E}$ is even equivalent to $P$ and hence in $\mathbb{P}_{f}(P) \cap \mathbb{P}_{e}$; see Theorem 2.2 of Frittelli (2000a). The standing assumption (1.1) is used here to appeal to Remark 2.1 of Frittelli (2000a); his Theorem 2.1 is stated only for bounded $X$, but extends readily to the locally bounded case.

The second useful consequence of (1.3) is related to $B$. For $B$ bounded from below or $B \in L^{1}(Q)$, Lemma 8 below proves the inequality

$$
E_{Q}[\gamma B] \leq H(Q \mid P)+\frac{1}{e} E\left[e^{\gamma B}\right] \quad \text { for } Q \ll P \text { and } \gamma>0
$$

Hence we have

$$
E_{Q}[\alpha B]<\infty \quad \text { for all } Q \in \mathbb{P}_{f}(P)
$$

under the assumption that

$$
B \text { is bounded from below and } E\left[e^{\alpha B}\right]<\infty \text {. }
$$


Note that in connection with $(0.1),(1.7)$ is a very natural assumption. Boundedness from below means that there is a uniform upper limit on the amount one can gain from the liability $B$. (Very often, $B$ models a payoff one has to make and is thus even nonnegative.) Moreover, the strategy $\vartheta \equiv 0$ ("do nothing") should always be in $\Theta$ and (1.7) then guarantees that the expected exponential utility of the outcome of total inactivity is not $-\infty$. In other words, the payoff $B$ is neither too good to be true nor so bad that doing nothing leads to a prohibitive punishment.

To define possible choices for the space $\Theta$ of permitted integrands, we now assume (1.7) and define a probability measure $P_{B}$ equivalent to $P$ by

$$
\frac{d P_{B}}{d P}:=c_{B} e^{\alpha B} \quad \text { with } c_{B}^{-1}:=E\left[e^{\alpha B}\right] \in(0, \infty)
$$

(This change of measure will allow us to essentially get rid of $B$.) Because $B$ is bounded from below, we obtain for $Q \ll P$

$$
H(Q \mid P)=E_{Q}\left[\log \frac{d Q}{d P_{B}}+\log c_{B}+\alpha B\right]=H\left(Q \mid P_{B}\right)+\log c_{B}+E_{Q}[\alpha B]
$$

so that in particular $H(Q \mid P)<\infty$ implies that $H\left(Q \mid P_{B}\right)<\infty$ due to (1.6). But our subsequent arguments also require the converse implication and we therefore strengthen (1.7) to the assumption that

$$
B \text { is bounded from below and } E\left[e^{(\alpha+\varepsilon) B}\right]<\infty \text { for some } \varepsilon>0 \text {. }
$$

If we then use (1.5) with $\gamma:=\varepsilon$ and for $P_{B}$ instead of $P$, we see that $H\left(Q \mid P_{B}\right)<\infty$ implies that $E_{Q}[\varepsilon B]<\infty$, hence $E_{Q}[\alpha B]<\infty$ and thus also $H(Q \mid P)<\infty$ by (1.8). In summary, (1.9) ensures that

$$
\mathbb{P}_{f}(P)=\left\{Q \in \mathbb{P}_{a} \mid H(Q \mid P)<\infty\right\}=\left\{Q \in \mathbb{P}_{a} \mid H\left(Q \mid P_{B}\right)<\infty\right\}=: \mathbb{P}_{f}\left(P_{B}\right)
$$

and we can simply write $\mathbb{P}_{f}$ from now on. In addition, we see that (1.3) or (1.4) transfer from $P$ to $P_{B}$ and vice versa in the sense that if these assumptions are satisfied with $P$ as reference measure, they also hold if we replace $P$ by $P_{B}$, and vice versa. We then denote under (1.4) by $Q^{E, B}$ the unique element of $\mathbb{P}_{f} \cap \mathbb{P}_{e}$ that minimizes $H\left(Q \mid P_{B}\right)$ over all $Q \in \mathbb{P}_{f}$ and call this the minimal $P_{B}$-entropy martingale measure. (Actually, the notation should be $Q^{E, P_{B}}$, but this is too heavy.) Then we can define

$$
\begin{aligned}
& \Theta_{1}:=\left\{\vartheta \in L(X) \mid e^{-\alpha G_{T}(\vartheta)} \in L^{1}\left(P_{B}\right) \text { and } G(\vartheta) \text { is a } Q^{E, B} \text {-martingale }\right\}, \\
& \Theta_{2}:=\left\{\vartheta \in L(X) \mid e^{-\alpha G_{T}(\vartheta)} \in L^{1}\left(P_{B}\right) \text { and } G(\vartheta) \text { is a } Q \text {-martingale for all } Q \in \mathbb{P}_{f}\right\}, \\
& \left.\Theta_{3}:=\{\vartheta \in L(X) \mid G(\vartheta) \text { is bounded (uniformly in } t \text { and } \omega)\right\} .
\end{aligned}
$$

It is evident that $\Theta_{3} \subseteq \Theta_{2} \subseteq \Theta_{1}$. For comparison purposes, we also introduce the space 


$$
\left.\Theta_{4}:=\{\vartheta \in L(X) \mid G(\vartheta) \text { is bounded from below (uniformly in } t \text { and } \omega)\right\} \text {. }
$$

Clearly, $\Theta_{3} \subseteq \Theta_{4}$, but there are no inclusions between $\Theta_{4}$ and either $\Theta_{1}$ or $\Theta_{2}$ in any generality.

Remark. The space $\Theta_{3}$ is obviously easiest to define, but also rather small; proving a duality result with $\Theta_{3}$ will therefore need more work. From a practical point of view, $\Theta_{3}$ is also important because any numerical approach will always employ some finite approximation and thus work with bounded processes. $\Theta_{1}$ has the advantage that it allows an easy proof (see section 2) for the corresponding duality and in addition contains the solution of the utility maximization problem in (1.2). On the other hand, $\Theta_{1}$ has the conceptual drawback that its very definition seems to anticipate the solution for (1.2). Finally, the intermediate space $\Theta_{2}$ is the natural analogue of a space used by Gouriéroux/Laurent/Pham (1998) in the context of an $L^{2}$-problem and is by its definition well adapted to duality formulations. However, it is difficult to verify for a given $\vartheta$ if it is in $\Theta_{2}$.

To state our first result, we need one additional preparation. We recall that under (1.1) and (1.4), the density of $Q^{E}$ with respect to $P$ has the form

$$
Z_{T}^{E}:=\frac{d Q^{E}}{d P}=c_{E} e^{G_{T}\left(\vartheta^{E}\right)}
$$

for some constant $c_{E}>0$ and some $\vartheta^{E} \in L(X)$ such that $G\left(\vartheta^{E}\right)$ is a $Q^{E}$-martingale; see Corollary 2.1 of Frittelli (2000a) and Proposition 3.2 and the proof of Theorem 4.13 in Grandits/ Rheinländer (1999). Taking logarithms and expectations under $Q^{E}$, we could write explicitly

$$
\log c_{E}=H\left(Q^{E} \mid P\right)
$$

In exactly the same way, we obtain

$$
Z_{T}^{E, B}:=\frac{d Q^{E, B}}{d P_{B}}=c_{E, B} e^{G_{T}\left(\vartheta^{E, B}\right)}
$$

for some constant $c_{E, B}>0$ and some $\vartheta^{E, B} \in L(X)$ such that $G\left(\vartheta^{E, B}\right)$ is a $Q^{E, B}$-martingale. Note that $-\frac{1}{\alpha} \vartheta^{E, B}=: \bar{\vartheta}$ is in $\Theta_{1}$ because

$$
e^{-\alpha G_{T}(\bar{\vartheta})}=e^{G_{T}\left(\vartheta^{E, B}\right)}=c_{E, B}^{-1} Z_{T}^{E, B}
$$

is obviously in $L^{1}\left(P_{B}\right)$.

Theorem 1. Assume (1.1), (1.4) and (1.9). Then

$$
\sup _{\vartheta \in \Theta_{1}} E\left[1-e^{-\alpha\left(c+G_{T}(\vartheta)-B\right)}\right]=1-\exp \left(-\inf _{Q \in \mathbb{P}_{f}}\left(H(Q \mid P)+\alpha c-E_{Q}[\alpha B]\right)\right)
$$


and the sup and inf are attained by $-\frac{1}{\alpha} \vartheta^{E, B} \in \Theta_{1}$ and $Q^{E, B} \in \mathbb{P}_{f} \cap \mathbb{P}_{e}$ respectively.

Our next two results need an additional assumption.

Definition. Let $Q \ll R$ be probability measures and denote by $Z_{t}^{Q \mid R}:=E_{R}\left[\frac{d Q}{d R} \mid \mathcal{F}_{t}\right]$ for $t \in[0, T]$ the density process of $Q$ with respect to $R$. We say that $Q$ satisfies the reverse Hölder inequality $R_{\mathrm{L} \log \mathrm{L}}(R)$ if there is a constant $C \in(0, \infty)$ such that

$$
E_{R}\left[\frac{Z_{T}^{Q \mid R}}{Z_{\tau}^{Q \mid R}} \log \frac{Z_{T}^{Q \mid R}}{Z_{\tau}^{Q \mid R}} \mid \mathcal{F}_{\tau}\right] \leq C \quad \text { for all stopping times } \tau \leq T
$$

(Note that with $\frac{0}{0}:=1$, the above ratios are well-defined by the maximum principle for supermartingales.)

Theorem 2. Assume (1.1), (1.4), (1.9) and

(1.13) there is some $Q \in \mathbb{P}_{f} \cap \mathbb{P}_{e}$ satisfying the reverse Hölder inequality $R_{\mathrm{L} \log \mathrm{L}}\left(P_{B}\right)$.

Then

$$
\sup _{\vartheta \in \Theta_{2}} E\left[1-e^{-\alpha\left(c+G_{T}(\vartheta)-B\right)}\right]=1-\exp \left(-\inf _{Q \in \mathbb{P}_{f}}\left(H(Q \mid P)+\alpha c-E_{Q}[\alpha B]\right)\right)
$$

and the sup and inf are attained by $-\frac{1}{\alpha} \vartheta^{E, B} \in \Theta_{2}$ and $Q^{E, B} \in \mathbb{P}_{f} \cap \mathbb{P}_{e}$ respectively.

Theorem 3. Assume (1.1), (1.4), (1.9) and (1.13). Then

$$
\sup _{\vartheta \in \Theta_{3}} E\left[1-e^{-\alpha\left(c+G_{T}(\vartheta)-B\right)}\right]=1-\exp \left(-\inf _{Q \in \mathbb{P}_{f}}\left(H(Q \mid P)+\alpha c-E_{Q}[\alpha B]\right)\right)
$$

and the inf is attained by $Q^{E, B} \in \mathbb{P}_{f} \cap \mathbb{P}_{e}$.

The proofs of all three theorems will be given later.

Remark. As mentioned before, a number of recent papers have more or less independently studied problems similar to ours. We try to give here a short overview to put the present paper into perspective.

Bellini/Frittelli (2000) focus on a duality as in (1.2) with a bounded contingent claim, the space $\Theta_{4}$ of admissible strategies and a general utility function $U$ defined on a subset of $\mathbb{R}$. (We have $U(x)=1-e^{-\alpha x}$.) They give sufficient conditions on $U$ for the duality to hold and the infimum over $\mathbb{P}$ to be attained by some $Q^{*}$. This $Q^{*}$ is then called a minimax 
martingale measure. For our case of exponential utility, their results give the duality (1.2) with $\Theta_{4}$ and without the assumption (1.13), under the sole assumptions (1.1) and (1.4). But a direct comparison with our Theorem 1 is not possible since $\Theta_{1}$ and $\Theta_{4}$ are not comparable, and our Theorem 3 has the additional assumption (1.13) but provides because of $\Theta_{3} \subseteq \Theta_{4}$ also a stronger result (and deals with some unbounded claims).

Davis (2000) is discussed at the end of subsection 4.3.

Frittelli (2000b) builds on the results from Bellini/Frittelli (2000) to construct a valuation for contingent claims by a utility indifference argument. He uses the duality to compute the resulting pricing functionals more explicitly and relates them to generalized distances between probability measures and to minimax martingale measures. The only overlap with our paper is the explicit formula (4.6) in subsection 4.2 for the exponential utility indifference price.

Goll/Rüschendorf (2001) study minimax and minimal distance martingale measures with their main focus on a general characterization of the latter for $f$-divergence distances. They provide a unified and general framework for the connections between expected utility maximization as primal optimization problems over portfolios/strategies, and $f$-projections arising from dual distance minimization problems over martingale measures. In particular, they describe optimal strategies in terms of minimax martingale measures and give a number of explicit examples for computations of these.

Rouge/El Karoui (2000) define a valuation for contingent claims by a utility indifference approach similar to the one in Frittelli (2000b). For the case of an exponential utility function $U(x)=1-e^{-\alpha x}$, they prove a duality result similar to ours under the assumption of a Brownian filtration; this is needed because they work with backward stochastic differential equations. Their space of strategies is not comparable to any of our $\Theta_{i}$. The main focus of their paper is on the properties of the resulting pricing rule, and one result which we also obtain in subsection 4.2 for our framework is concerned with the risk-averse limit as $\alpha \rightarrow \infty$.

Schachermayer (2000) obtains a duality result of the type (1.2) for a locally bounded semimartingale $X$ with a more general utility function $U$ defined on $\mathbb{R}$, but without a contingent claim $B$. He starts with the space $\Theta_{4}$ and then maximizes $E[U(G)]$ over the $L^{1}(P)$-closure of the set of all random variables $U(G)$ such that $G$ can be superreplicated by some $\vartheta \in \Theta_{4}$. The main result is then a duality between this primal problem and the dual problem of minimizing a functional $V$, conjugate to $U$, over the space $\mathbb{P}_{a}$ of ALMMs. (A similar problem is solved in Cvitanić/Schachermayer/Wang (2001)). If the latter infimum is attained by some ELMM (i.e., in some $Q \in \mathbb{P}_{e}$ ), Schachermayer (2000) also shows that the solution to the primal problem is actually attained by the final wealth of some self-financing strategy. For the special case where $U$ is exponential, this implies our Theorem 1 , and in general, it is also very similar to the results of Bellini/Frittelli (2000), Goll/Rüschendorf (2001) and Xia/Yan (2000). A comparison to our Theorems 2 and 3 is not possible.

Xia/Yan (2000) also show how to construct an optimal strategy for a utility maximization problem from a solution of the dual problem over martingale measures. They give some ex- 
plicit examples if $X$ is driven by a Lévy process with time-dependent coefficients; their utility functions do not include the exponential. The special case of an exponential Lévy process with constant coefficients has been studied in Kallsen (2000); he gives explicit solutions in terms of the semimartingale characteristics, and his examples include exponential utility.

In summary, our paper is different in two aspects. A first difference is that we have an exogenous contingent claim $B$ satisfying milder conditions than previously imposed in the literature. As section 2 shows, the presence of $B$ is technically a minor point which can (in our approach) be dealt with by a measure transformation. The more important difference is in the goal of our paper. Since we are interested in hedging, we want to focus on strategies and on what can be achieved by them. Our primary concern is therefore the choice of the strategy space $\Theta$, and our results in Theorems 2 and 3 say that under suitable assumptions, several choices lead to the same value in the basic duality. This is a robustness property which is complementary to all of the existing literature and thus constitutes a major new contribution. For additional recent results in this direction (obtained after this paper was submitted), see also Schachermayer (2001).

\section{Reducing the problem}

Our goal in this section is twofold. We want to show that it is enough to consider the case $c=0, \alpha=1, B \equiv 0$ and we give some auxiliary results used in the proofs in the next sections.

First of all, the constant 1 and the factor $e^{-\alpha c}$ can be omitted from both sides of (1.2). Writing LHS and RHS for the remaining left- and right-hand sides of (1.2), this leaves us with

$$
L H S=\sup _{\vartheta \in \Theta}-E\left[e^{-\alpha G_{T}(\vartheta)+\alpha B}\right]=c_{B}^{-1} \sup _{\vartheta \in \Theta}-E_{P_{B}}\left[e^{-\alpha G_{T}(\vartheta)}\right]=c_{B}^{-1} \sup _{\vartheta \in \Theta}-E_{P_{B}}\left[e^{-G_{T}(\alpha \vartheta)}\right]
$$

and

$$
\begin{aligned}
R H S & =-\exp \left(-\inf _{Q \in \mathbb{P}_{f}}\left(H(Q \mid P)-E_{Q}[\alpha B]\right)\right) \\
& =-c_{B}^{-1} \exp \left(-\inf _{Q \in \mathbb{P}_{f}} H\left(Q \mid P_{B}\right)\right) \\
& =-c_{B}^{-1} e^{-H\left(Q^{E, B} \mid P_{B}\right)}
\end{aligned}
$$

by (1.8) and the definition of $Q^{E, B}$. Moreover, the definitions of $\Theta_{i}, i=1,2,3$, make it clear that $\vartheta$ is in $\Theta_{i}$ if and only if $\alpha \vartheta$ is in $\Theta_{i}^{\prime}$ with

$$
\begin{aligned}
& \Theta_{1}^{\prime}:=\left\{\vartheta \in L(X) \mid e^{-G_{T}(\vartheta)} \in L^{1}\left(P_{B}\right) \text { and } G(\vartheta) \text { is a } Q^{E, B} \text {-martingale }\right\}, \\
& \Theta_{2}^{\prime}:=\left\{\vartheta \in L(X) \mid e^{-G_{T}(\vartheta)} \in L^{1}\left(P_{B}\right) \text { and } G(\vartheta) \text { is a } Q \text {-martingale for all } Q \in \mathbb{P}_{f}\right\}, \\
& \Theta_{3}^{\prime}:=\Theta_{3} .
\end{aligned}
$$


Hence it is enough to prove Theorem $i, i=1,2,3$, in the formulation obtained by replacing $\Theta_{i}$ by $\Theta_{i}^{\prime}, P$ by $P_{B}, \alpha$ by $1, c$ by 0 and $B$ by 0 throughout the formulations. For $i=1$, this is already enough preparation.

Proof of Theorem 1. As argued above, we have to show that

$$
\sup _{\vartheta \in \Theta_{1}^{\prime}}-E_{B}\left[e^{-G_{T}(\vartheta)}\right]=-e^{-H\left(Q^{E, B} \mid P_{B}\right)}
$$

where we write for brevity $E_{B}$ for expectation under $P_{B}$. Since $H\left(Q^{E, B} \mid P_{B}\right)=\log c_{E, B}$ by (1.11), the RHS of (2.1) equals $-c_{E, B}^{-1}$. For any $\vartheta \in \Theta_{1}^{\prime}$, (1.12) and Jensen's inequality yield

$$
E_{B}\left[e^{-G_{T}(\vartheta)}\right]=c_{E, B}^{-1} E_{Q^{E, B}}\left[e^{-G_{T}\left(\vartheta^{E, B}\right)-G_{T}(\vartheta)}\right] \geq c_{E, B}^{-1}
$$

since $G\left(\vartheta^{E, B}\right)$ and $G(\vartheta)$ are $Q^{E, B}$-martingales. Moreover, the remark before Theorem 1 shows that $-\vartheta^{E, B}$ is in $\Theta_{1}^{\prime}$ and

$$
E_{B}\left[e^{G_{T}\left(\vartheta^{E, B}\right)}\right]=c_{E, B}^{-1}
$$

Thus the LHS of (2.1) also equals $-c_{E, B}^{-1}$ and this proves both (2.1) and the assertion that sup and inf are attained by $-\vartheta^{E, B}$ and $Q^{E, B}$ respectively. Because the inf in (2.1) is over $\Theta_{1}^{\prime}$, the inf over $\Theta_{1}$ is attained at $-\frac{1}{\alpha} \vartheta^{E, B}$ and this completes the proof.

q.e.d.

If we look more closely at the above argument, we see that it uses only structural properties in the following sense. Suppose we start with any probability $R$ equivalent to $P$ such that we can find a minimal $R$-entropy martingale measure $Q^{E, R}$. If we then define $\Theta_{1}^{\prime}(R)$ by replacing $P_{B}$ with $R$ and $Q^{E, B}$ with $Q^{E, R}$, the only properties we need to prove the $R$-version of (2.1) are the entropy-minimality of $Q^{E, R}$ (with respect to $R$ ) and the structure of the density $\frac{d Q^{E, R}}{d R}$ as in (1.12). Because $R \equiv P$ does possess these properties, we could therefore equally well prove the result for $R \equiv P$ and then use the above transfer mechanism to get the $P_{B}$-version we really want. Put differently, the change of measure from $P$ to $P_{B}$ has the effect that "we can argue as if $B$ were identically 0". Exactly the same thing happens with Theorems 2 and 3 and we shall exploit this fact by arguing throughout the rest of this and the next section with $P$ instead of $P_{B}$. This greatly simplifies the notation.

The next lemma is a variation of similar results from Delbaen/Monat/Schachermayer/ Schweizer/Stricker (1997) and Grandits/Krawczyk (1998). Yuri Kabanov has pointed out that we could formulate this more generally, but we refrain from doing so to avoid extra notation; see however Kabanov/Stricker (2001b) for related results. Let us also remark that 
Lemma 4 allows to give a shorter argument for Corollary 4.15 of Grandits/Rheinländer (1999), thus answering a question raised in the introduction of Rheinländer (1999).

Lemma 4. Assume (1.1) and (1.4). Then the condition there is some $Q \in \mathbb{P}_{f}$ satisfying the reverse Hölder inequality $R_{\mathrm{L} \log \mathrm{L}}(P)$ implies that the minimal P-entropy martingale measure $Q^{E}$ satisfies the reverse Hölder inequality $R_{\mathrm{L} \log \mathrm{L}}(P)$.

The best constant in (2.3) can be taken less than or equal to the best constant in (2.2).

Proof. Take $Q$ from (2.2) and denote by $Z^{Q}$ and $Z^{E}$ the respective density processes, with respect to $P$, of $Q$ and of $Q^{E}$. Due to $(2.2)$, there is some constant $K \in(0, \infty)$ such that

$$
E\left[\frac{Z_{T}^{Q}}{Z_{\tau}^{Q}} \log \frac{Z_{T}^{Q}}{Z_{\tau}^{Q}} \mid \mathcal{F}_{\tau}\right] \leq K \quad \text { for all stopping times } \tau \leq T .
$$

If $Q^{E}$ does not satisfy $R_{\mathrm{L} \log \mathrm{L}}(P)$ with a constant $\leq K$, there exist $\varepsilon>0$, a stopping time $\sigma \leq T$ and a set $A \in \mathcal{F}_{\sigma}$ with $P[A]>0$ such that

$$
E\left[\frac{Z_{T}^{E}}{Z_{\sigma}^{E}} \log \frac{Z_{T}^{E}}{Z_{\sigma}^{E}} \mid \mathcal{F}_{\sigma}\right] \geq K+\varepsilon \quad \text { on } A \text {. }
$$

Define

$$
\widetilde{Z}_{t}:=I_{\{t<\sigma\}} Z_{t}^{E}+I_{\{t \geq \sigma\}}\left(I_{A} \frac{Z_{t}^{Q}}{Z_{\sigma}^{Q}} Z_{\sigma}^{E}+I_{A^{c}} Z_{t}^{E}\right) \quad \text { for } t \in[0, T]
$$

and note that $\widetilde{Z}$ is the density process with respect to $P$ of some $\widetilde{Q} \in \mathbb{P}_{a}$. Moreover,

$$
\widetilde{Z}_{T}=I_{A} Z_{\sigma}^{E} \frac{Z_{T}^{Q}}{Z_{\sigma}^{Q}}+I_{A^{c}} Z_{T}^{E}
$$

implies that

$$
\widetilde{Z}_{T} \log \widetilde{Z}_{T}=I_{A^{c}} Z_{T}^{E} \log Z_{T}^{E}+I_{A}\left(Z_{\sigma}^{E} \frac{Z_{T}^{Q}}{Z_{\sigma}^{Q}} \log \frac{Z_{T}^{Q}}{Z_{\sigma}^{Q}}+\frac{Z_{T}^{Q}}{Z_{\sigma}^{Q}} Z_{\sigma}^{E} \log Z_{\sigma}^{E}\right)
$$

and therefore

$$
\begin{aligned}
& E\left[\widetilde{Z}_{T} \log \widetilde{Z}_{T} \mid \mathcal{F}_{\sigma}\right]-E\left[Z_{T}^{E} \log Z_{T}^{E} \mid \mathcal{F}_{\sigma}\right] \\
& =I_{A}\left(Z_{\sigma}^{E} E\left[\frac{Z_{T}^{Q}}{Z_{\sigma}^{Q}} \log \frac{Z_{T}^{Q}}{Z_{\sigma}^{Q}} \mid \mathcal{F}_{\sigma}\right]+Z_{\sigma}^{E} \log Z_{\sigma}^{E}-E\left[Z_{T}^{E} \log Z_{T}^{E} \mid \mathcal{F}_{\sigma}\right]\right) \\
& =I_{A} Z_{\sigma}^{E}\left(E\left[\frac{Z_{T}^{Q}}{Z_{\sigma}^{Q}} \log \frac{Z_{T}^{Q}}{Z_{\sigma}^{Q}} \mid \mathcal{F}_{\sigma}\right]-E\left[\frac{Z_{T}^{E}}{Z_{\sigma}^{E}} \log \frac{Z_{T}^{E}}{Z_{\sigma}^{E}} \mid \mathcal{F}_{\sigma}\right]\right) \\
& \leq-\varepsilon I_{A} Z_{\sigma}^{E}
\end{aligned}
$$


by (2.4) and (2.5). Hence taking expectations yields $H(\widetilde{Q} \mid P)-H\left(Q^{E} \mid P\right)<0$ which contradicts the fact that $Q^{E}$ is the minimal $P$-entropy martingale measure.

q.e.d.

For our next result, we have to introduce the process

$$
\bar{Z}_{t}:=\exp \left(E_{Q^{E}}\left[\log Z_{T}^{E} \mid \mathcal{F}_{t}\right]\right)=c_{E} \exp \left(G_{t}\left(\vartheta^{E}\right)\right) \quad, \quad 0 \leq t \leq T
$$

the second equality uses $(1.10)$ and that $G\left(\vartheta^{E}\right)$ is a $Q^{E}$-martingale. We again denote by $Z^{E}$ the density process of $Q^{E}$ with respect to $P$.

Lemma 5. Assume (1.1) and (1.4). If (2.2) is satisfied, then we have

$$
Z_{t}^{E} \leq \bar{Z}_{t} \leq C Z_{t}^{E} \quad, \quad 0 \leq t \leq T
$$

for some constant $C \in(0, \infty)$. More precisely: the left inequality is always true, the right one is equivalent to (2.2).

Proof. By Lemma 4, (2.2) is equivalent to (2.3), and since the Bayes rule and (2.6) yield

$$
\log \bar{Z}_{t}=E\left[\frac{Z_{T}^{E}}{Z_{t}^{E}} \log \frac{Z_{T}^{E}}{Z_{t}^{E}}+\frac{Z_{T}^{E}}{Z_{t}^{E}} \log Z_{t}^{E} \mid \mathcal{F}_{t}\right]=E\left[\frac{Z_{T}^{E}}{Z_{t}^{E}} \log \frac{Z_{T}^{E}}{Z_{t}^{E}} \mid \mathcal{F}_{t}\right]+\log Z_{t}^{E}
$$

we see that $(2.3)$ is equivalent to the right inequality in (2.7). On the other hand, $1 / Z^{E}$ is the density process of $P$ with respect to $Q^{E}$ so that Jensen's inequality always gives

$$
\log Z_{t}^{E}=-\log E_{Q^{E}}\left[\frac{d P}{d Q^{E}} \mid \mathcal{F}_{t}\right] \leq E_{Q^{E}}\left[\log Z_{T}^{E} \mid \mathcal{F}_{t}\right]=\log \bar{Z}_{t}
$$

q.e.d.

Lemma 6. Assume (1.1) and (1.4). For every $Q \in \mathbb{P}_{f}, G\left(\vartheta^{E}\right)$ is then a $Q$-supermartingale, and even a $Q$-martingale if (2.2) is satisfied.

Proof. Since $\mathbb{P}_{f} \cap \mathbb{P}_{e} \neq \emptyset$ by (1.4), we may assume (by replacing $Q$ with $\frac{1}{2}\left(Q+Q^{E}\right)$ if necessary) that $Q$ is equivalent to $P$, hence in $\mathbb{P}_{f} \cap \mathbb{P}_{e}$. Thus $Q, P$ and $Q^{E}$ are all equivalent. According to Theorem 2.2 of Csiszár (1975), we have

$$
H(Q \mid P) \geq H\left(Q \mid Q^{E}\right)+H\left(Q^{E} \mid P\right)
$$

and since $Q \in \mathbb{P}_{f}$, this implies

$$
H\left(Q \mid Q^{E}\right)<\infty \text { and } H(Q \mid P)<\infty
$$


Lemma 2 of Barron (1985) states that if $\left(\mathcal{G}_{n}\right)_{n \in \mathbb{N}}$ is a filtration on $(\Omega, \mathcal{F}, P)$ and $R \ll P$ is another probability measure on $(\Omega, \mathcal{F})$, the densities

$$
U_{n}:=\left.\frac{d R}{d P}\right|_{\mathcal{G}_{n}}=E\left[\frac{d R}{d P} \mid \mathcal{G}_{n}\right] \quad, \quad n \in \mathbb{N}
$$

satisfy

$$
E_{R}\left[\sup _{n \in \mathbb{N}}\left|\log U_{n}\right|\right] \leq C(H(R \mid P)+1)
$$

It is straightforward to extend this result to a continuous-time filtration and so if $Z^{1}$ denotes the density process of $Q^{E}$ with respect to $Q$ and $Z^{2}$ the density process of $Q$ with respect to $P,(2.8)$ implies that

$$
\sup _{0 \leq t \leq T}\left|\log Z_{t}^{i}\right| \in L^{1}(Q) \quad \text { for } i=1,2
$$

Because the density process of $Q^{E}$ with respect to $P$ is $Z^{E}=Z^{1} Z^{2}$, we obtain

$$
\sup _{0 \leq t \leq T}\left|\log Z_{t}^{E}\right| \in L^{1}(Q)
$$

Now $L:=G\left(\vartheta^{E}\right)=\log \bar{Z}-\log c_{E}$ by (2.6) and so the (always valid) left inequality in (2.7) yields $L_{t} \geq C+\log Z_{t}^{E}$, hence

$$
\sup _{0 \leq t \leq T} L_{t}^{-} \in L^{1}(Q)
$$

by (2.9). Since $L=G\left(\vartheta^{E}\right)=\int \vartheta^{E} d X$ and $X$ is a local $Q$-martingale, we conclude from Proposition 3.3 in Ansel/Stricker (1994) and Fatou's lemma that $L$ is a local $Q$-martingale and a $Q$-supermartingale. If we have (2.2), we can by Lemma 5 also use the right inequality in (2.7) to obtain $L_{t} \leq C+\log Z_{t}^{E}$ and therefore

$$
\sup _{0 \leq t \leq T}\left|L_{t}\right| \in L^{1}(Q)
$$

again by (2.9). Thus $L=G\left(\vartheta^{E}\right)$ is then a true $Q$-martingale.

q.e.d.

The next result will be used in section 4 .

Lemma 7. Assume (1.4). Then $\left\{\frac{d Q}{d P} \mid Q \in \mathbb{P}_{f} \cap \mathbb{P}_{x}\right\}$ is $L^{1}(P)$-dense in $\left\{\frac{d Q}{d P} \mid Q \in \mathbb{P}_{x}\right\}$ for $x \in\{a, e\}$.

Proof. In this and even greater generality, Lemma 7 has recently been proved by Kabanov/Stricker (2001a); see their Corollary 1.3. We give here a proof that assumes in addition 
that the filtration $\mathbb{F}$ is continuous, i.e., all $(P, \mathbb{F})$-martingales are continuous. Fix $Q \in \mathbb{P}_{x}$ and denote by $Z$ its density process with respect to $P$. Due to (1.4), the density process $Z^{E}$ with respect to $P$ of the minimal entropy martingale measure $Q^{E}$ is strictly positive and so the stopping times

$$
\tau_{n}:=\inf \left\{t \in[0, T] \mid Z_{t} \geq n \text { or } Z_{t}^{E} \leq \frac{1}{n}\right\} \wedge T
$$

increase to $T$ stationarily $P$-a.s. For each $n$, the process $Z^{(n)}:=Z I_{\left[0, \tau_{n}[[\right.}+\frac{Z_{\tau_{n}}}{Z_{\tau_{n}}^{E}} Z^{E} I_{\left.\left.\llbracket \tau_{n}, T\right]\right]}$ is the density process of some $R_{n} \in \mathbb{P}_{x}$. Moreover, $Z_{T}^{(n)}$ converges for $n \rightarrow \infty$ to $Z_{T} P$-a.s. and in $L^{1}(P)$ since $Z_{T}$ and all $Z_{T}^{(n)}$ are nonnegative and satisfy $\lim _{n \rightarrow \infty} E\left[Z_{T}^{(n)}\right]=1=E\left[Z_{T}\right]$. Thus it only remains to show that each $R_{n}$ is in $\mathbb{P}_{f}$. But $Z$ and $Z^{E}$ are both continuous and so we get

$$
Z_{T}^{(n)} \log Z_{T}^{(n)}=\frac{Z_{\tau_{n}}}{Z_{\tau_{n}}^{E}} Z_{T}^{E} \log Z_{T}^{E}+Z_{T}^{E} \frac{Z_{\tau_{n}}}{Z_{\tau_{n}}^{E}} \log \frac{Z_{\tau_{n}}}{Z_{\tau_{n}}^{E}} \leq n^{2} Z_{T}^{E} \log ^{+} Z_{T}^{E}+Z_{T}^{E} n^{2} \log ^{+} n^{2}
$$

by the definition of $\tau_{n}$. Taking expectations and using $x \log ^{+} x \leq x \log x+e^{-1}$ yields

$$
H\left(R_{n} \mid P\right) \leq C_{n}\left(1+H\left(Q^{E} \mid P\right)\right)<\infty
$$

and completes the proof.

q.e.d.

For completeness, we prove the following useful estimate.

Lemma 8. Let $Q$ be a probability measure and $B$ a random variable that is uniformly bounded from below or in $L^{1}(Q)$. Then

$$
E_{Q}[B] \leq H(Q \mid P)+\frac{1}{e} E\left[e^{B}\right]
$$

Proof. The left-hand side is well-defined in $(-\infty, \infty]$ by assumption and we can obviously assume that $Q \ll P$. Since $x \log x \geq-\frac{1}{e}$ implies for $x=\frac{a}{b}$ that

$$
a \log b \leq a \log a+\frac{b}{e} \quad \text { for } a \geq 0, b>0
$$

we get the result by choosing $a=\frac{d Q}{d P}$ and $b=e^{B}$ and taking expectations under $P$. 


\section{Proof of Theorems 2 and 3}

In this section, we prove Theorems 2 and 3.

Proof of Theorem 2. Since $-\frac{1}{\alpha} \vartheta^{E, B}$ is in $\Theta_{2}$ by Lemma 6, the same calculation as in the argument for Theorem 1 shows that $-\frac{1}{\alpha} \vartheta^{E, B}$ attains the sup in (1.14) and that

$$
\sup _{\vartheta \in \Theta_{2}} E\left[1-e^{-\alpha\left(c+G_{T}(\vartheta)-B\right)}\right]=1-\exp \left(-\inf _{Q \in \mathbb{P}_{f}}\left(H(Q \mid P)+\alpha c-E_{Q}[\alpha B]\right)\right) .
$$

Because we already know from the proof of Theorem 1 that the above inf is attained by $Q^{E, B}$, the proof is complete.

q.e.d.

Proof of Theorem 3. As argued in section 2, we have to show that

$$
\sup _{\vartheta \in \Theta_{3}^{\prime}}-E_{P_{B}}\left[e^{-G_{T}(\vartheta)}\right]=-e^{-H\left(Q^{E, B} \mid P_{B}\right)}
$$

or equivalently

$$
I_{3}:=\inf _{\vartheta \in \Theta_{3}^{\prime}} E_{P_{B}}\left[e^{-G_{T}(\vartheta)}\right]=e^{-H\left(Q^{E, B} \mid P_{B}\right)} .
$$

Under the assumptions of Theorem 3, Theorem 1 holds and gives

$$
e^{-H\left(Q^{E, B} \mid P_{B}\right)}=E_{P_{B}}\left[e^{-G_{T}\left(-\vartheta^{E, B}\right)}\right]=\inf _{\vartheta \in \Theta_{1}^{\prime}} E_{P_{B}}\left[e^{-G_{T}(\vartheta)}\right] \leq I_{3}
$$

because $\Theta_{1}^{\prime} \supseteq \Theta_{3}^{\prime}$. Hence it is enough to prove that there exists a sequence $\left(\vartheta^{n}\right)_{n \in \mathbb{N}}$ in $\Theta_{3}^{\prime}=\Theta_{3}$ such that

$$
\lim _{n \rightarrow \infty} E_{P_{B}}\left[e^{-G_{T}\left(\vartheta^{n}\right)}\right]=E_{P_{B}}\left[e^{-G_{T}\left(-\vartheta^{E, B}\right)}\right] .
$$

But this follows from Lemma 9 below, applied to $P_{B}$ instead of $P$.

q.e.d.

The only missing piece is now provided by the following approximation result.

Lemma 9. Assume (1.1), (1.4) and (2.2) and recall from (1.10) the process $\vartheta^{E}$ in the representation of the minimal $P$-entropy measure $Q^{E}$. Then there exists a sequence $\left(\vartheta^{n}\right)_{n \in \mathbb{N}}$ in $\Theta_{3}$ such that

$$
\lim _{n \rightarrow \infty} E\left[e^{-G_{T}\left(\vartheta^{n}\right)}\right]=E\left[e^{G_{T}\left(\vartheta^{E}\right)}\right]
$$


Proof. Define $U:=e^{G\left(\vartheta^{E}\right)}=c_{E}^{-1} \bar{Z}$ by $(2.6)$ and write $Y_{t}^{*}:=\sup _{0 \leq s \leq t}\left|Y_{s}\right|$ for any process $Y$ on $[0, T]$ and any $t \in[0, T]$. Recall that $Z^{E}$ is the density process of $Q^{E}$ with respect to $P$.

1) For any $N \in \mathbb{I}$, define $\xi^{N}:=\vartheta^{E} I_{\left\{\left|\vartheta^{E}\right| \leq N\right\}}$. Since $\vartheta^{E}$ is in $L(X)$, the sequence $\left(G\left(\xi^{N}\right)\right)_{N \in \mathbb{N}}$ converges to $G\left(\vartheta^{E}\right)$ in the semimartingale topology and so $U^{N}:=e^{G\left(\xi^{N}\right)}$ converges to $U$ in the semimartingale topology by Proposition 4 of Émery (1979). Hence $\left(U^{N}-U\right)_{T}^{*}$ converges to 0 P-a.s. along a subsequence (again labelled by $N$ ) so that the stopping times

$$
\tau_{N}:=\inf \left\{t \in[0, T] \mid U_{t}^{N} \geq 2 U_{t}\right\} \wedge T
$$

increase to $T$ stationarily $P$-a.s. The process $\psi^{N}:=-\xi^{N} I_{\left.] 0, \tau_{N}\right]}$ is therefore in $L(X)$ and bounded by $N$ and

$$
e^{-G_{T}\left(\psi^{N}\right)}=\exp \left(G_{\tau_{N}}\left(\xi^{N}\right)\right)=U_{\tau_{N}}^{N} \longrightarrow U_{T}=e^{G_{T}\left(\vartheta^{E}\right)} \quad P \text {-a.s. as } N \rightarrow \infty \text {. }
$$

We want to obtain convergence in $L^{1}(P)$ and so we need an estimate from above.

2) To get a bound on $U_{\tau_{N}}^{N}$ uniformly in $N$, we first note that

$$
\left(U^{N}\right)_{\tau_{N}}^{*} \leq\left|\Delta U_{\tau_{N}}^{N}\right|+\sup _{0 \leq t<\tau_{N}} U_{t}^{N} \leq\left|\Delta U_{\tau_{N}}^{N}\right|+2 U_{\tau_{N}}^{*}
$$

because $U_{t}^{N}<2 U_{t}$ for $t<\tau_{N}$. Moreover, the definition of $\xi^{N}$ implies that

$$
\begin{aligned}
\frac{U_{\tau_{N}}^{N}}{U_{\tau_{N}-}^{N}}-1 & =\exp \left(\Delta G_{\tau_{N}}\left(\xi^{N}\right)\right)-1 \\
& =I_{\left\{\left|\vartheta_{\tau_{N}}^{E}\right| \leq N\right\}}\left(\exp \left(\Delta G_{\tau_{N}}\left(\vartheta^{E}\right)\right)-1\right) \\
& =I_{\left\{\left|\vartheta_{\tau_{N}}^{E}\right| \leq N\right\}}\left(\frac{U_{\tau_{N}}}{U_{\tau_{N}}-1}\right)
\end{aligned}
$$

so that by the definition of $\tau_{N}$

$$
\left|\Delta U_{\tau_{N}}^{N}\right|=U_{\tau_{N}-}^{N}\left|\frac{U_{\tau_{N}}^{N}}{U_{\tau_{N}-}^{N}}-1\right| \leq 2 U_{\tau_{N}-}\left|\frac{U_{\tau_{N}}}{U_{\tau_{N}-}}-1\right| \leq 2 U_{\tau_{N}}+2 U_{\tau_{N}-}
$$

Hence we obtain

$$
\left(U^{N}\right)_{\tau_{N}}^{*} \leq 6 U_{\tau_{N}}^{*} \leq 6 U_{T}^{*} \leq C\left(Z^{E}\right)_{T}^{*}
$$

for some constant $C$ by Lemma 5 . But $Z^{E}$ is a $P$-martingale with

$$
E\left[Z_{T}^{E} \log ^{+} Z_{T}^{E}\right] \leq \frac{1}{e}+H\left(Q^{E} \mid P\right)<\infty
$$


hence $\left(Z^{E}\right)_{T}^{*}$ is in $L^{1}(P)$ and therefore

$$
\sup _{N} U_{\tau_{N}}^{N} \leq 6 U_{T}^{*} \in L^{1}(P)
$$

Combining this with (3.2) yields

$$
\lim _{N \rightarrow \infty} E\left[e^{-G_{T}\left(\psi^{N}\right)}\right]=E\left[e^{G_{T}\left(\vartheta^{E}\right)}\right]
$$

3) Because $G\left(\psi^{N}\right)$ need not be bounded, $\psi^{N}$ need not be in $\Theta_{3}$ and (3.5) is not yet good enough for (3.1). So we exploit (1.1) and the boundedness of $\psi^{N}$ in order to stop $X$ and $G\left(\psi^{N}\right)$ to get them both bounded and to construct $\vartheta^{n}$. More precisely, (1.1) allows us to find stopping times $\left(\sigma_{m}\right)_{m \in \mathbb{N}}$ increasing stationarily to $T$ such that $X^{\sigma_{m}}$ is bounded by a constant $K_{m}$, say, for each $m$. Fix $N$ and define

$$
\varrho_{m}:=\sigma_{m} \wedge \inf \left\{t \in[0, T]|| G_{t}\left(\psi^{N}\right) \mid>m\right\} \wedge T
$$

and $\vartheta^{m, N}:=\psi^{N} I_{\left.\left.]] 0, \varrho_{m}\right]\right]}$. Then $\left(\varrho_{m}\right)_{m \in \mathbb{N}}$ increases stationarily to $T$ and $\vartheta^{m, N}$ is in $L(X)$ for each $m$ and even in $\Theta_{3}$ because

$$
\begin{aligned}
\left(G\left(\vartheta^{m, N}\right)\right)_{T}^{*} & =\left(G\left(\psi^{N}\right)\right)_{\varrho_{m}}^{*} \\
& \leq\left|\Delta G_{\varrho_{m}}\left(\psi^{N}\right)\right|+\sup _{0 \leq t<\varrho_{m}}\left|G_{t}\left(\psi^{N}\right)\right| \\
& \leq\left|\psi_{\varrho_{m}}^{N}\right|\left|\Delta X_{\varrho_{m}}\right|+m \\
& \leq 2 N K_{m}+m
\end{aligned}
$$

by the definition of $\varrho_{m}$ and since $\psi^{N}$ is bounded by $N$. Moreover,

$$
\lim _{m \rightarrow \infty} e^{-G_{T}\left(\vartheta^{m, N}\right)}=\lim _{m \rightarrow \infty} e^{-G_{\varrho m}\left(\psi^{N}\right)}=e^{-G_{T}\left(\psi^{N}\right)} \quad P \text {-a.s. }
$$

and because

$$
e^{-G_{T}\left(\vartheta^{m, N}\right)}=e^{-G_{\varrho_{m}}\left(\psi^{N}\right)}=\exp \left(G_{\varrho_{m} \wedge \tau_{N}}\left(\xi^{N}\right)\right)=U_{\varrho_{m} \wedge \tau_{N}}^{N} \leq\left(U^{N}\right)_{\tau_{N}}^{*} \leq 6 U_{T}^{*} \quad \text { for all } m
$$

by (3.3), we conclude from (3.4) that

$$
\lim _{m \rightarrow \infty} E\left[e^{-G_{T}\left(\vartheta^{m, N}\right)}\right]=E\left[e^{-G_{T}\left(\psi^{N}\right)}\right] \quad \text { for each } N
$$

Combining this with (3.5) and using a diagonalization procedure to construct a single sequence $\left(\vartheta^{n}\right)_{n \in \mathbb{N}}$ completes the proof.

q.e.d. 


\section{Applications and examples}

In this section, we give some applications and examples related to our basic duality result. Because maximizing exponential utility has been studied extensively in a number of papers, we focus here except in subsection 4.3 on situations where the duality itself plays a key role. In specific examples, one needs of course the density of the minimal entropy martingale measure and perhaps the optimal strategy in more explicit form, but this is not our goal here. We refer instead to Chan (1999), Davis (2000) or Grandits/Rheinländer (1999) for various results in that direction.

\subsection{A dual characterization of the minimal martingale measure}

Suppose that the process $X$ is continuous and that $\mathbb{P}_{e} \neq \emptyset$. Then it is well known that $X$ satisfies the structure condition (SC): there exist an $\mathbb{R}^{d}$-valued continuous local $P$-martingale $M$ null at 0 and an $\mathbb{R}^{d}$-valued predictable process $\lambda \in L_{\text {loc }}^{2}(M)$ such that the canonical $P$-decomposition of $X$ is $X=X_{0}+M+\int d\langle M\rangle \lambda$. The process $\widehat{K}:=\int \lambda^{\operatorname{tr}} d\langle M\rangle \lambda=$ $\left\langle-\int \lambda d M\right\rangle$ is called the mean-variance tradeoff process of $X$. If the local $P$-martingale $\widehat{Z}:=\mathcal{E}\left(-\int \lambda d M\right)>0$ is a true $P$-martingale, $\widehat{P}$ defined by

$$
\frac{d \widehat{P}}{d P}:=\widehat{Z}_{T}
$$

is an ELMM for $X$, i.e., in $\mathbb{P}_{e}$. This $\widehat{P}$ is called the minimal martingale measure. In Föllmer/ Schweizer (1991), $\widehat{P}$ is characterized within a certain class of martingale measures as the minimizer of the functional $j(Q):=H(Q \mid P)-\frac{1}{2} E_{Q}\left[\widehat{K}_{T}\right]$. Our general duality result allows us to write down a new primal hedging problem whose dual is precisely the minimization of $j$; see Theorem 10 below.

A well-known sufficient condition for $\widehat{Z}$ to be a true $P$-martingale is Novikov's condition

$$
E\left[\exp \left(\frac{1}{2} \widehat{K}_{T}\right)\right]<\infty
$$

Now fix $\alpha>0$ and $c \in \mathbb{R}$ and consider the contingent claim $B:=\frac{1}{2 \alpha} \widehat{K}_{T}$. Because $\widehat{K}$ is nonnegative, it is evident that (1.7) is satisfied if and only if (4.1) holds. To apply Theorem 1 , however, we have to impose (1.9) and this translates here as

$$
E\left[\exp \left(\frac{1+\varepsilon}{2} \widehat{K}_{T}\right)\right]<\infty \quad \text { for some } \varepsilon>0
$$

This implies (4.1), hence $\widehat{P} \in \mathbb{P}_{e}$, and so $\mathbb{P}_{f} \cap \mathbb{P}_{e}$ is then nonempty if $H(\widehat{P} \mid P)<\infty$. 
At this point, it is natural to ask whether the Novikov condition (4.1) already implies $H(\widehat{P} \mid P)<\infty$. This is not true and the argument goes as follows. Take any $Q \approx P$ with $Q=P$ on $\mathcal{F}_{0}$ and write its density process $Z>0$ as $Z=\mathcal{E}(N)$ for some local $P$-martingale $N$ null at 0 . It is clear that $H(Q \mid P)<\infty$ is equivalent to $Z_{T} \log Z_{T} \in L^{1}(P)$, and if $Z$ is continuous, this is equivalent to $Z_{T}^{*}:=\sup _{0 \leq t \leq T} Z_{t} \in L^{1}(P)$, i.e., to $Z$ being in $\mathcal{H}^{1}(P)$; see the proof of Theorem 1.9 in Kazamaki (1994). So the above problem boils down to the question whether $E\left[\exp \left(\frac{1}{2}\langle N\rangle_{T}\right)\right]<\infty$ for a continuous local $P$-martingale $N$ null at 0 implies that $\mathcal{E}(N)$ is in $\mathcal{H}^{1}(P)$. This is false as shown by Example 3.1 in Kazamaki (1994).

Fortunately, our stronger assumption (4.2) does guarantee that $H(\widehat{P} \mid P)<\infty$. In fact, Yan (1980) shows that a continuous local martingale $N$ which is null at 0 and satisfies $E\left[\exp \left(\frac{\gamma}{2}\langle N\rangle_{T}\right)\right]<\infty$ for some $\gamma>1$ has $Z=\mathcal{E}(N) \in \mathcal{H}^{r}(P)$, i.e., $Z_{T}^{*} \in L^{r}(P)$, for some $r=r(\gamma)>1$; see also Remark 1.2 of Kazamaki (1994). This implies that $Z_{T} \log Z_{T} \in L^{1}(P)$, hence the conclusion.

Theorem 10. Suppose that $X$ is continuous, $\mathbb{P}_{e} \neq \emptyset$ and (4.2) is satisfied. Then the minimal martingale measure $\widehat{P}$ corresponds via duality to the problem of hedging with exponential utility a particular multiple of the total mean-variance tradeoff $\widehat{K}_{T}$. More precisely: For any $\alpha>0$ and $c \in \mathbb{R}$, the optimization problems

$$
\text { for } B:=\frac{1}{2 \alpha} \widehat{K}_{T} \text {, maximize } E\left[1-e^{-\alpha\left(c+G_{T}(\vartheta)-B\right)}\right] \text { over } \vartheta \in \Theta_{1}
$$

and

$$
\text { for } B:=\frac{1}{2 \alpha} \widehat{K}_{T}, \text { minimize } 1-\exp \left(-\left(H(Q \mid P)+\alpha c-E_{Q}[\alpha B]\right)\right) \text { over } Q \in \mathbb{P}_{f}
$$

have the same value and the minimal martingale measure $\widehat{P}$ solves (4.4).

Proof. Note that $H(\widehat{P} \mid P)<\infty$ due to (4.2); see the discussion before Theorem 10 . The statement that (4.3) and (4.4) have the same value is just a reformulation of the key duality in Theorem 1. To solve (4.4), one obviously has to find

$$
\inf _{Q \in \mathbb{P}_{f}}\left(H(Q \mid P)-\frac{1}{2} E_{Q}\left[\widehat{K}_{T}\right]\right)=\inf \left\{H(Q \mid P)-\frac{1}{2} E_{Q}\left[\widehat{K}_{T}\right] \mid Q \in \mathbb{P}_{a} \text { with } H(Q \mid P)<\infty\right\} .
$$

Due to (4.2) and Lemma 8, we have

$$
\left\{Q \in \mathbb{P}_{a} \text { with } H(Q \mid P)<\infty\right\} \subseteq\left\{Q \in \mathbb{P}_{a} \text { with } E_{Q}\left[\widehat{K}_{T}\right]<\infty\right\}
$$

and if $Q \in \mathbb{P}_{a}$ satisfies $E_{Q}\left[\widehat{K}_{T}\right]<\infty$ but $H(Q \mid P)=+\infty$, then $H(Q \mid P)-\frac{1}{2} E_{Q}\left[\widehat{K}_{T}\right]=+\infty$. Hence we obtain

$$
\inf _{Q \in \mathbb{P}_{f}}\left(H(Q \mid P)-\frac{1}{2} E_{Q}\left[\widehat{K}_{T}\right]\right)=\inf \left\{H(Q \mid P)-\frac{1}{2} E_{Q}\left[\widehat{K}_{T}\right] \mid Q \in \mathbb{P}_{a} \text { with } E_{Q}\left[\widehat{K}_{T}\right]<\infty\right\} .
$$


But the last infimum is under our assumptions attained by $\widehat{P}$; see Theorem 5 of Schweizer (1995) and observe that the assumption $Q=P$ on $\mathcal{F}_{0}$ is not used in the proofs there.

One novel feature of Theorem 10 is that we obtain the minimal martingale measure for the first time as the solution of one of a pair of well-defined and dual optimization problems. This was first recognized in Samperi (1999) in a diffusion context. Note also that the minimal martingale measure $\widehat{P}$ coincides with the minimal entropy martingale measure $Q^{E}$ if $E_{Q}\left[\widehat{K}_{T}\right]$ does not depend on $Q \in \mathbb{P}_{f}$. This is trivially satisfied if $\widehat{K}_{T}$ is deterministic. It also happens in a possibly multidimensional Markovian diffusion model with $X$ given by a stochastic differential equation of the form

$$
d X_{t}=\mu\left(t, X_{t}\right) d t+\sigma\left(t, X_{t}\right) d W_{t}
$$

see Miyahara (1996) for more details.

\subsection{Risk-averse asymptotics}

Let us define

$$
J_{\alpha}(c, B):=\sup _{\vartheta \in \Theta} E\left[1-e^{-\alpha\left(c+G_{T}(\vartheta)-B\right)}\right]
$$

as the maximal expected utility associated to the initial capital $c$ and the final payoff obligation $B$. Following Hodges/Neuberger (1989), we can then define the utility indifference price $p_{\alpha}(c, B) \in \mathbb{R}$ by the implicit equation

$$
J_{\alpha}(c, 0)=J_{\alpha}\left(c+p_{\alpha}(c, B), B\right) .
$$

In terms of maximal expected utility, we thus view $p_{\alpha}(c, B)$ as an adequate initial compensation for taking on an additional risk of $B$. Since $\alpha>0$ is the risk aversion parameter of the exponential utility function appearing in $(1.2)$, we can ask what happens to $p_{\alpha}(c, B)$ in the totally risk-averse limit as $\alpha$ tends to infinity. This question has also been studied by Rouge/El Karoui (2000) in the context of a Brownian filtration by using backward stochastic differential equations and dynamic programming methods, and part of their Theorem 5.2 has the same conclusion as our Corollary 12 below. For recent general results on $p_{\alpha}(c, B)$, see also Becherer (2001).

Let us first use (1.2) to rewrite (4.5) as

$$
\begin{aligned}
p_{\alpha}(c, B) & =p_{\alpha}(B) \\
& =\sup _{Q \in \mathbb{P}}\left(E_{Q}[B]-\frac{1}{\alpha} H(Q \mid P)\right)-\sup _{Q \in \mathbb{P}}\left(-\frac{1}{\alpha} H(Q \mid P)\right) \\
& =v_{\alpha}(B)-v_{\alpha}(0)
\end{aligned}
$$


with

$$
v_{\alpha}(B):=\sup _{Q \in \mathbb{P}}\left(E_{Q}[B]-\frac{1}{\alpha} H(Q \mid P)\right)=\frac{1}{\alpha} \log \inf _{\vartheta \in \Theta} E\left[e^{-\alpha\left(G_{T}(\vartheta)-B\right)}\right] .
$$

Note that we have used (1.2) in its abstract formulation with unspecified $\Theta, \mathbb{P}$. We apply this below with $\Theta_{1}, \mathbb{P}_{f}$ and write $\Theta_{1}(\alpha)$ to emphasize the fact that this space depends on $\alpha$.

Proposition 11. Assume (1.1), (1.4) and (1.9) for all $\alpha>0$. Then

$$
\lim _{\alpha \rightarrow \infty} v_{\alpha}(B)=\sup _{Q \in \mathbb{P}_{f} \cap \mathbb{P}_{e}} E_{Q}[B]
$$

Proof. For $\Theta=\Theta_{1}(\alpha)$, Theorem 1 and (4.7) show that $v_{\alpha}(B)$ is increasing in $\alpha$ for each fixed $B$ and so the left-hand side of (4.8) is

$$
\lim _{\alpha \rightarrow \infty} v_{\alpha}(B)=\sup _{\alpha>0} v_{\alpha}(B)=\sup _{Q \in \mathbb{P}_{f}} \sup _{\alpha>0}\left(E_{Q}[B]-\frac{1}{\alpha} H(Q \mid P)\right)=\sup _{Q \in \mathbb{P}_{f}} E_{Q}[B]
$$

Since $\mathbb{P}_{f} \cap \mathbb{P}_{e} \neq \emptyset$ by (1.4) and $B$ is bounded from below, it is easy to show that

$$
\sup _{Q \in \mathbb{P}_{f}} E_{Q}[B]=\sup _{Q \in \mathbb{P}_{f} \cap \mathbb{P}_{e}} E_{Q}[B]
$$

and this implies (4.8).

The next result shows that the limit of the utility indifference price $p_{\alpha}(B)$ as $\alpha \rightarrow \infty$ is the super-replication price for $B$; this generalizes a result of Rouge/El Karoui (2000) to the case of a general filtration.

Corollary 12. Assume (1.1), (1.4) and (1.9) for all $\alpha>0$. Then

$$
\lim _{\alpha \rightarrow \infty} p_{\alpha}(B)=\sup _{Q \in \mathbb{P}_{e}} E_{Q}[B]
$$

Proof. Since $p_{\alpha}(B)=v_{\alpha}(B)-v_{\alpha}(0)$ and $\lim _{\alpha \rightarrow \infty} v_{\alpha}(0)=0$, it is clear from (4.8) that it only remains to show that $\sup _{Q \in \mathbb{P}_{e}} E_{Q}[B] \leq \sup _{Q \in \mathbb{P}_{f} \cap \mathbb{P}_{e}} E_{Q}[B]$. Because $B$ is bounded from below due to (1.9), Lemma 7 yields for each fixed $Q \in \mathbb{P}_{e}$ a sequence $\left(R_{n}\right)_{n \in \mathbb{N}}$ in $\mathbb{P}_{f} \cap \mathbb{P}_{e}$ such that

$$
E_{Q}[B \wedge m]=\lim _{n \rightarrow \infty} E_{R_{n}}[B \wedge m] \leq \sup _{Q \in \mathbb{P}_{f} \cap \mathbb{P}_{e}} E_{Q}[B]
$$


for each $m \in \mathbb{N}$. Hence the assertion follows by monotone convergence.

Our second result shows that with increasing risk aversion, the optimal strategy for hedging with exponential utility tends to behave like super-replication if one can afford it. For a precise formulation, let

$$
c^{*}:=\sup _{Q \in \mathbb{P}_{e}} E_{Q}[B]
$$

be the super-replication price for $B$ and denote by $\vartheta_{\alpha}^{*}=-\frac{1}{\alpha} \vartheta^{E, B}$ the solution of

$$
\text { maximize } E\left[1-e^{-\alpha\left(c+G_{T}(\vartheta)-B\right)}\right] \text { over } \vartheta \in \Theta_{1} \text {. }
$$

Note that $\vartheta_{\alpha}^{*}$ does not depend on $c$ in (4.9).

Theorem 13. Assume (1.1), (1.4) and (1.9) for all $\alpha>0$. Then

$$
\lim _{\alpha \rightarrow \infty}\left(c^{*}+G_{T}\left(\vartheta_{\alpha}^{*}\right)-B\right)^{-}=0 \quad \text { in } L^{1}(P) .
$$

Proof. If we set $U_{\alpha}:=c^{*}+G_{T}\left(\vartheta_{\alpha}^{*}\right)-B$, then Theorem 1 yields

$$
\log E\left[e^{-\alpha U_{\alpha}}\right]=-\inf _{Q \in \mathbb{P}_{f}}\left(H(Q \mid P)+\alpha c^{*}-E_{Q}[\alpha B]\right)=: R H S .
$$

Thanks to $(1.9), c^{*} \in(-\infty,+\infty]$ and Lemma 8 therefore guarantees that the quantity $H(Q \mid P)+\alpha c^{*}-E_{Q}[\alpha B]$ is always well-defined in $(-\infty,+\infty]$ for any $Q \in \mathbb{P}_{f}$. For $c^{*}=+\infty$, $R H S$ is $-\infty$ and for $c^{*}<\infty$, one easily proves from (1.4) and the definition of $c^{*}$ that $R H S \leq 0$. Jensen's inequality gives

$$
E\left[U_{\alpha}^{-}\right] \leq \frac{1}{\alpha} \log E\left[e^{\alpha U_{\alpha}^{-}}\right] \leq \frac{1}{\alpha} \log \left(1+E\left[e^{-\alpha U_{\alpha}}\right]\right)
$$

and this implies the assertion by (4.10).

q.e.d.

\subsection{Markovian models}

In this subsection, we take a look at the optimization problem

$$
\text { maximize } E\left[1-e^{-\alpha\left(c+G_{T}(\vartheta)-B\right)}\right] \text { over all } \vartheta \in \Theta
$$

within a Markovian framework. This type of situation is often encountered in applications. Our main goal is to point out some links to existing literature; the computations we present 
are purely formal and it would be interesting to see them worked out in more detail. Some results in that direction have recently been obtained by Davis (2000).

We start with an $\mathbb{R}^{m}$-valued Markov diffusion process $S$ which represents some basic state variables in our model. $S$ is given by a stochastic differential equation

$$
d S_{t}=\widetilde{a}\left(t, S_{t}\right) d t+\widetilde{b}\left(t, S_{t}\right) d W_{t} \quad \text { under } P
$$

for suitable vector- and matrix-valued functions $\widetilde{a}, \widetilde{b}$. We assume that $X_{t}=c\left(t, S_{t}\right)$ for some function $c$ and consider a contingent claim of the form $B=g\left(S_{T}\right)$. Since everything except $\vartheta$ in (4.11) then only depends on the Markov process $S$, it seems plausible that Markovian strategies of the form $\vartheta_{t}=h\left(t, S_{t}\right)$ should be sufficient to solve (4.11). If we write $X$ as

$$
d X_{t}=a\left(t, S_{t}\right) d t+b\left(t, S_{t}\right) d W_{t}
$$

we thus obtain an optimization problem of the form

$$
\operatorname{minimize} E\left[\exp \left(\alpha g\left(S_{T}\right)-\alpha \int_{0}^{T} h^{\operatorname{tr}}\left(u, S_{u}\right)\left(a\left(u, S_{u}\right) d u+b\left(u, S_{u}\right) d W_{u}\right)\right)\right]
$$

over all suitable functions $h(t, s)$.

This is not (yet) a usual optimal control problem since the objective function contains a stochastic integral term $\int \ldots d W$. To apply standard results, we want to eliminate this term by a Girsanov transformation; this was suggested in Samperi (1998) and is similar to the approach in Laurent/Pham (1999).

So fix a function $h$ and consider the exponential local $P$-martingale

$$
Z^{h}=\mathcal{E}\left(-\alpha \int h^{\operatorname{tr}} b d W\right)=\exp \left(-\alpha \int h^{\operatorname{tr}} b d W-\frac{1}{2} \alpha^{2} \int\left|h^{\operatorname{tr}} b\right|^{2} d u\right)
$$

with all functions evaluated in $\left(u, S_{u}\right)$. Then our objective function can be written as

$$
\begin{aligned}
& E\left[\exp \left(\alpha g\left(S_{T}\right)-\alpha \int_{0}^{T}\left(h^{\operatorname{tr}}\left(u, S_{u}\right) a\left(u, S_{u}\right) d u+h^{\operatorname{tr}}\left(u, S_{u}\right) b\left(u, S_{u}\right) d W_{u}\right)\right)\right] \\
& =E\left[Z_{T}^{h} \exp \left(\alpha g\left(S_{T}\right)-\alpha \int_{0}^{T}\left(h^{\operatorname{tr}} a-\frac{1}{2} \alpha\left|h^{\operatorname{tr}} b\right|^{2}\right)\left(u, S_{u}\right) d u\right)\right]
\end{aligned}
$$

If $Z^{h}$ is a $P$-martingale, it defines by Girsanov's theorem a probability measure $P^{h}$ equivalent to $P$ under which $W^{h}=W+\alpha \int h^{\operatorname{tr}}\left(u, S_{u}\right) b\left(u, S_{u}\right) d u$ is a Brownian motion. Since

$$
d S_{t}=\widetilde{a}\left(t, S_{t}\right) d t+\widetilde{b}\left(t, S_{t}\right) d W_{t}=\left(\widetilde{a}-\alpha \widetilde{b} b^{\operatorname{tr}} h\right)\left(t, S_{t}\right) d t+\widetilde{b}\left(t, S_{t}\right) d W_{t}^{h},
$$


we see that

$S$ has under $P^{h}$ the same distribution as $Y$ under $P$

if we define $Y$ by

$$
d Y_{t}=\left(\widetilde{a}-\alpha \widetilde{b} b^{\operatorname{tr}} h\right)\left(t, Y_{t}\right) d t+\widetilde{b}\left(t, Y_{t}\right) d W_{t} \quad \text { under } P .
$$

Combining this with (4.13), we conclude that (4.12) is equivalent to

$$
\operatorname{minimize} E\left[\exp \left(\alpha g\left(Y_{T}\right)-\alpha \int_{0}^{T}\left(h^{\mathrm{tr}} a-\frac{1}{2} \alpha\left|h^{\mathrm{tr}} b\right|^{2}\right)\left(u, Y_{u}\right) d u\right)\right]
$$

over all suitable functions $h(t, y)$

with $Y$ given by (4.14). In contrast to (4.12), (4.15) is now a standard optimal control problem. The corresponding Hamilton-Jacobi-Bellman equation for the associated value function $V:[0, T] \times \mathbb{R}^{m}$ is

$$
\inf _{h \in \mathbb{R}^{d}}\left(V_{t}+\frac{1}{2} \sum_{i, j=1}^{d}\left(\widetilde{b} b^{\operatorname{tr}}\right)_{i j} V_{y_{i} y_{j}}+\left(\widetilde{a}-\alpha \widetilde{b} b^{\operatorname{tr}} h\right)^{\operatorname{tr}} V_{y}-\alpha\left(h^{\operatorname{tr}} a-\frac{1}{2} \alpha\left|h^{\operatorname{tr}} b\right|^{2}\right) V\right)=0
$$

with boundary condition

$$
V(T, y)=e^{\alpha g(y)} \quad \text { for } y \in \mathbb{R}^{d}
$$

where subscripts stand for partial derivatives and $V_{y}$ is the gradient of $V$. For any given problem, one can now try to solve this explicitly; the optimal strategy is then obtained as the minimizer $h^{*}\left(t, S_{t}\right)$ in (4.16) and can be expressed in terms of $V$.

Example. For the case where $S=X$ is a one-dimensional geometric Brownian motion with constant coefficients and $g \equiv 0,(4.11)$ was solved by Pliska (1986) as an application of more general results on utility maximization for complete markets.

Example. In Browne (1995), a problem of the type (4.11) was solved by optimal control arguments, but with a different setup and reformulation than used here. Browne (1995) took $X$ as a one-dimensional geometric Brownian motion with constant coefficients and $B=$ $-U_{T}$ for a second Brownian motion $U$ with drift and correlated with $X$. His filtration was generated by $X$ (and not like here by the state variable process $S=(X U)^{\text {tr }}$ ) and the resulting Hamilton-Jacobi-Bellman equation was solved explicitly. The nice feature of this example is that one has an explicit solution for an incomplete market with a genuinely random contingent claim $B$. 
Example. In the one-dimensional case $m=d=1$, (4.16) can be rewritten as

$$
\inf _{h \in \mathbb{R}}\left(V_{t}+\frac{1}{2} \widetilde{b}^{2} V_{y y}+(\widetilde{a}-\alpha \widetilde{b} b h) V_{y}-\left(\alpha h a-\frac{1}{2} \alpha^{2} h^{2} b^{2}\right) V\right)=0
$$

Minimizing over $h$ yields

$$
h^{*}\left(t, S_{t}\right)=\frac{1}{\alpha}\left(\frac{a}{b^{2}}+\frac{\widetilde{b}}{b} \frac{V_{y}}{V}\right)\left(t, S_{t}\right)
$$

and inserting this into (4.17) leads to the nonlinear partial differential equation

$$
V_{t}-\frac{1}{2} \frac{a^{2}}{b^{2}} V+\frac{1}{2} \widetilde{b}^{2}\left(V_{y y}-\frac{V_{y}^{2}}{V}\right)+\left(\widetilde{a}-\frac{a \widetilde{b}}{b}\right) V_{y}=0
$$

for $V(t, y)$ with the same boundary condition as before. In the special case where $g \equiv$ const. and the ratio $\frac{a}{b}$ does not depend on $y$, the solution is easily seen to be

$$
V(t, y)=\text { const. } \exp \left(-\frac{1}{2} \int_{t}^{T} \frac{a^{2}}{b^{2}}(u) d u\right)=\text { const. } e^{-\frac{1}{2}\left(\widehat{K}_{T}-\widehat{K}_{t}\right)}
$$

and the optimal strategy is then

$$
\vartheta_{t}^{*}=h^{*}\left(t, S_{t}\right)=\frac{1}{\alpha} \frac{a\left(t, S_{t}\right)}{b^{2}\left(t, S_{t}\right)}
$$

This is due to Samperi (1998). Since the mean-variance tradeoff process $\widehat{K}=\int \frac{a^{2}}{b^{2}}(u) d u$ is here deterministic, it is not very surprising that we can find an explicit solution.

Example. A recent paper by Davis (2000) studies the problem of finding the utility indifference price defined by (4.5) in the case where $B$ is an option written on one geometric Brownian motion which is imperfectly correlated with the tradable asset $X$ given by another geometric Brownian motion. In this situation, Davis (2000) gives quasi-explicit formulae for the optimal strategy and the solution of the dual problem, both expressed in terms of the solution of a certain Hamilton-Jacobi-Bellman equation. Solving that equation explicitly appears difficult.

\subsection{How far can one go?}

While Theorem 1 is true under almost minimal conditions, Theorems 2 and 3 use in addition the reverse Hölder inequality assumption (1.13). In this subsection, we explore to some extent the question if this condition could be avoided. As a positive result, the next example shows 
that (1.13) is sufficient, but not necessary for the duality in Theorem 2 to hold. For the case where $B$ is bounded, the results of Bellini/Frittelli (2000) also show that (1.13) is not necessary for the infimum in (0.1) to be attained. A definitive answer to this question has recently been given by Kabanov/Stricker (2001b).

Example. Let $W=\left(W_{t}\right)_{t \geq 0}$ be a standard Brownian motion on $(\Omega, \mathcal{G}, P)$ and $\mathbb{G}=$ $\left(\mathcal{G}_{t}\right)_{0 \leq t \leq \infty}$ the $P$-augmentation of the natural filtration of $W$ with $\mathcal{G}_{\infty}=\bigvee_{t \geq 0} \mathcal{G}_{t}$. The stopping time $\tau:=\inf \left\{t \geq 0 \mid W_{t}=1\right\}$ then satisfies $\tau<\infty P$-a.s. and

$$
E[\tau]=+\infty
$$

Define the filtration $\mathbb{F}=\left(\mathcal{F}_{t}\right)_{0 \leq t \leq \infty}$ by $\mathcal{F}_{t}=\mathcal{G}_{t \wedge \tau}$ and take $\mathcal{F}=\mathcal{F}_{\infty}=\mathcal{G}_{\tau}$. The process $X=\left(X_{t}\right)_{0 \leq t \leq \infty}$ is given by

$$
X_{t}:=W_{t}^{\tau}-t \wedge \tau=W_{t \wedge \tau}-t \wedge \tau \quad, \quad 0 \leq t \leq \infty
$$

Then we claim that

$$
\text { the duality result (1.14) with } \Theta_{2} \text { holds }
$$

although

$$
\text { (1.13) is not satisfied. }
$$

The intuition behind this is very simple: Because there is a unique ELMM for $X$ on $\mathcal{F}=\mathcal{G}_{\tau}$, $\Theta_{1}$ and $\Theta_{2}$ coincide and there is no need for the assumption (1.13).

To prove (4.19), we first note that the martingale representation property of $W$ and the structure of $X$ imply that the density process $Z$ of any $Q \in \mathbb{P}_{e}$ must be of the form

$$
Z_{t}=\mathcal{E}\left(W^{\tau}\right)_{t}=\exp \left(W_{t \wedge \tau}-\frac{1}{2}(t \wedge \tau)\right) \quad, \quad 0 \leq t \leq \infty .
$$

Hence $\mathbb{P}_{e}=\{Q\}$ is a singleton and

$$
0 \leq H(Q \mid P)=E_{Q}\left[\log Z_{\infty}\right]=E_{Q}\left[1-\frac{1}{2} \tau\right]
$$

shows that $E_{Q}[\tau]<\infty$ and $H(Q \mid P)<\infty$. Thus (1.4) is satisfied, the minimal entropy martingale measure $Q^{E}$ exists and coincides with $Q$, we have $\Theta_{1}=\Theta_{2}$, and (4.19) holds with $B \equiv 0$. For readers puzzled by the fact that $E_{P}[\tau]=+\infty$ but $E_{Q}[\tau]<\infty$, we point out that $W$ has a positive drift under $Q$; it is thus easier for $W$ to reach 1 under $Q$ than under $P$.

We now turn to (4.20). Marc Yor has suggested to one of us a direct proof based on the explicit form of the conditional Laplace transform of $\tau$, but we give here an alternative 
abstract argument. If (1.13) were satisfied for $B \equiv 0, Q=Q^{E}$ would satisfy $R_{\mathrm{L} \log \mathrm{L}}(P)$. Thus Lemma 4.14 and Lemma 2.2 of Grandits/Rheinländer (1999) would imply that $Q$ also satisfies a so-called reverse Hölder inequality $R_{p}(P)$ for some $p>1$. (This is like $R_{\mathrm{L} \log \mathrm{L}}(P)$ with the function $x \log x$ replaced by $x^{p}$.) Proposition 5 of Doléans-Dade/Meyer (1979) would then in turn imply that $Z$ satisfies a so-called Muckenhoupt condition $A_{q}(P)$ for some $q>1$ and this would in particular imply that

$$
\frac{d P}{d Q}=\frac{1}{Z_{\tau}} \text { is in } L^{\varepsilon}(P) \text { for some } \varepsilon>0
$$

But this is false: Jensen's inequality gives

$$
E\left[\frac{1}{Z_{\tau}^{\varepsilon}}\right]=E\left[\exp \left(-\varepsilon+\frac{1}{2} \varepsilon \tau\right)\right] \geq \exp \left(-\varepsilon+\frac{1}{2} \varepsilon E[\tau]\right)=+\infty
$$

due to (4.18). This proves (4.20) and ends the example.

Acknowledgments. The research of FD is sponsored by Crédit Suisse. TR gratefully acknowledges financial support by the Deutsche Forschungsgemeinschaft through the Graduiertenkolleg "Stochastische Prozesse und probabilistische Analysis" at the Technical University of Berlin. DS thanks S.R.S. Varadhan for helpful conversations. MS thanks Dirk Becherer for useful discussions and gratefully acknowledges financial support by the Deutsche Forschungsgemeinschaft via the Sonderforschungsbereich 373 "Quantifikation und Simulation Ökonomischer Prozesse" at Humboldt University, Berlin. CS thanks Marc Yor for helpful conversations. We are all grateful to the referees and to Yuri Kabanov for a number of useful hints and suggestions.

All the usual disclaimers apply. The opinions and statements in the paper only reflect the authors' viewpoints. The sponsoring does not change this.

\section{References}

J.-P. Ansel and C. Stricker (1994), "Couverture des Actifs Contingents", Annales de l'Institut Henri Poincaré 30, 303-315

A. R. Barron (1985), "The Strong Ergodic Theorem for Densities: Generalized ShannonMcMillan-Breiman Theorem", Annals of Probability 13, 1292-1303

D. Becherer (2001), "Rational Hedging and Valuation with Utility-Based Preferences", $\mathrm{PhD}$ thesis, Technical University of Berlin 
F. Bellini and M. Frittelli (2000), "On the Existence of Minimax Martingale Measures", preprint 14/2000, University of Milano-Bicocca

A. Ben-Tal (1985), "The Entropic Penalty Approach to Stochastic Programming", Mathematics of Operations Research 10, 263-279

S. Browne (1995), "Optimal Investment Policies for a Firm with a Random Risk Process: Exponential Utility and Minimizing the Probability of Ruin", Mathematics of Operations Research 20, 937-958

T. Chan (1999), "Pricing Contingent Claims on Stocks Driven by Lévy Processes", Annals of Applied Probability 9, 504-528

I. Csiszár (1975), "I-Divergence Geometry of Probability Distributions and Minimization Problems", Annals of Probability 3, 146-158

J. Cvitanić, W. Schachermayer and H. Wang (2001), "Utility Maximization in Incomplete Markets with Random Endowment", Finance and Stochastics 5, 259-272

M. Davis (2000), "Optimal Hedging with Basis Risk", preprint, Imperial College, London

F. Delbaen, P. Monat, W. Schachermayer, M. Schweizer and C. Stricker (1997), "Weighted Norm Inequalities and Hedging in Incomplete Markets", Finance and Stochastics 1, 181-227

C. Doléans-Dade and P. A. Meyer (1979), "Inégalités de Normes avec Poids", Séminaire de Probabilités XIII, Lecture Notes in Mathematics 721, Springer, 313-331

M. Émery (1979), "Une Topologie sur l'Espace des Semimartingales", Séminaire de Probabilités XIII, Lecture Notes in Mathematics 721, Springer, 313-331

M. Frittelli (2000a), "The Minimal Entropy Martingale Measure and the Valuation Problem in Incomplete Markets", Mathematical Finance 10, 39-52

M. Frittelli (2000b), "Introduction to a Theory of Value Coherent with the No-Arbitrage Principle", Finance and Stochastics 4, 275-297

T. Goll and L. Rüschendorf (2001), "Minimax and Minimal Distance Martingale Measures and their Relationship to Portfolio Optimization", Finance and Stochastics 5, 557-581

C. Gouriéroux, J. P. Laurent and H. Pham (1998), "Mean-Variance Hedging and Numéraire", Mathematical Finance 8, 179-200

P. Grandits and L. Krawczyk (1998), "Closedness of Some Spaces of Stochastic Integrals", Séminaire de Probabilités XXXII, Lecture Notes in Mathematics 1686, Springer, 73-85 
P. Grandits and T. Rheinländer (1999), "On the Minimal Entropy Martingale Measure", preprint, Technical University of Berlin, to appear in Annals of Probability

S. D. Hodges and A. Neuberger (1989), "Optimal Replication of Contingent Claims under Transaction Costs", Review of Futures Markets 8, 222-239

Yu. Kabanov and C. Stricker (2001a), "On Equivalent Martingale Measures with Bounded Densities", Séminaire de Probabilités XXXV, Lecture Notes in Mathematics 1755, Springer, 139-148

Yu. Kabanov and C. Stricker (2001b), "On the Optimal Portfolio for the Exponential Utility Maximization: Remarks to the Six-Author Paper", preprint, Université de FrancheComté, Besançon, to appear in Mathematical Finance

J. Kallsen (2000), "Optimal Portfolios for Exponential Lévy Processes", Mathematical Methods of Operations Research 51, 357-374

N. Kazamaki (1994), "Continuous Exponential Martingales and BMO", Lecture Notes in Mathematics 1579, Springer

D. Kramkov and W. Schachermayer (1999), "The Asymptotic Elasticity of Utility Functions and Optimal Investment in Incomplete Markets", Annals of Applied Probability 9, 904-950

J. P. Laurent and H. Pham (1999), "Dynamic Programming and Mean-Variance Hedging", Finance and Stochastics 3, 83-110

Y. Miyahara (1996), "Canonical Martingale Measures of Incomplete Assets Markets", in: Probability Theory and Mathematical Statistics, Proceedings of the Seventh Japan-Russia Symposium, Tokyo 1995, 343-352

S. R. Pliska (1986), "A Stochastic Calculus Model of Continuous Trading: Optimal Portfolios", Mathematics of Operations Research 11, 371-382

T. Rheinländer (1999), "Optimal Martingale Measures and their Applications in Mathematical Finance", PhD thesis, Technical University of Berlin

R. Rouge and N. El Karoui (2000), "Pricing via Utility Maximization and Entropy", Mathematical Finance 10, 259-276

D. Samperi (1998), "Inverse Problems, Model Selection and Entropy in Derivative Security Pricing", PhD thesis, New York University

D. Samperi (1999), "On Entropy and Mean-Variance Hedging", preprint [unpublished]

D. Samperi (2000), "Model Selection Using Entropy and Geometry", preprint, Decision 
Synergy, New York

W. Schachermayer (2000), "Optimal Investment in Incomplete Markets when Wealth May Become Negative", preprint, Technical University of Vienna, to appear in Annals of Applied Probability

W. Schachermayer (2001), "How Potential Investments May Change the Optimal Portfolio for the Exponential Utility", preprint, Technical University of Vienna

M. Schweizer (1995), "On the Minimal Martingale Measure and the Föllmer-Schweizer Decomposition", Stochastic Analysis and Applications 13, 573-599

J. Xia and J.-A. Yan (2000), "Optimal Strategy for Utility Maximization in a Market Driven by a Lévy Process", preprint, Chinese Academy of Sciences, Beijing

J.-A. Yan (1980), "Criteria for the Uniform Integrability of Exponential Martingales", Acta Mathematica Sinica 23, 311-318 [in Chinese] 